(Aus dem Kaiserin Auguste Victoria-Haus, Reichsanstalt zur Bekämpfung der Säuglings- und Kleinkindersterblichkeit.)

\title{
Bedenken gegen die Verwendung der Begriffe Toxikose und Intoxikation als Krankheitsbezeichnung.
}

\author{
Von \\ L. Langstein und H. Langer.
}

(Eingegangen am 30. November 1921.)

Der Begriff der Intoxikation hat durch die verschiedenartige Auslegung, die er gegenwärtig findet, wobei er bald zur Bezeichnung einer Krankheitsphase, bald zur Bezeichnung der Krankheit selbst verwendet wird, die Klarheit verloren, die für eine voraussetzungslose Verständigung unentbehrlich ist. Diese Unklarheit ist durch die Einführung des Begriffes der Toxikose nur gesteigert worden, zumal über eine Abgrenzung der beiden Begriffe untereinander keine Finigung besteht.

So kommt es, daß die vergleichende Kritik bei der Gegenüberstellung etwa von therapeutischen Ergebnissen auf große Schwierigkeiten stößt, die nur durch ausführliche Schilderung klinischer Einzelheiten überwunden werden können.

Finkelstein hat in seiner grundlegenden Darstellung der Ernährungsstörungen des Säuglings diese als Einheit aufgefaßt: Aus dem Stadium der Bilanzstörung entsteht das der Dyspepsie, von ihm aus gabelt sich der Weg und führt entweder zum Stadium der Intoxikation oder Dekomposition - je nach der Art der Ernährung. Die Phase der Intoxikation sah Finkelstein in einem Enterokatarrh, der durch eine Folge von Symptomen eine besondere Charakterisierung erfährt. Unter diesen steht in erster Reihe die Veränderung der Psyche des Kindes, die Trübung des Sensoriums. Unlösbar mit der Bewußtseinstrübung sollten verknüpft sein: Fieber, große Atmung, Leukocytose, Albuminurie, Cylindrurie, Glykosurie, Kollaps und Gewichtssturz. Das Vorhandensein sämtlicher Symptome war damals für Finkelstein die Voraussetzung für die Diagnose der Intoxikation.

Im Gegensatz zu ihm entwickelten Czerny-Keller den Begriff der Intoxikation aus dem weiten Begriff der Toxikose. Sie ordneten den ganzen Komplex der akuten Ernährungsstörungen unter die ex infektione. Sie betrachteten als eine Hauptgruppe jene, bei denen die 
Zersetzung der Nahrung als ätiologischer Faktor wirksam ist, und erblickten die Möglichkeit der Unterordnung unter den Infektionsbegriff darin, daß Bakterien an der Zersetzung beteiligt sind. Mit dem Beiwort ,alimentär" wollten sie zum Ausdruck bringen, daß die Nahrung einzig und allein die Schädigung auslöst, wobei diese nicht eine Folge falscher Zusammensetzung der Nahrung, sondern der Veränderung (Zersetzung) der Nahrung ist.

Finkelstein hingegen hatte bei seiner Schilderung ausschließlich dann von Intoxikation gesprochen, wenn eine durch Veränderung des Sensoriums gekennzeichneten Form des Enterokatarrhs gegeben ist, bei dem dann bestimmte stets vorhandene Begleitsymptome die differentialdiagnostische Abgrenzung sichern. Für Finkelstein war also der Intoxikationsbegriff eine Zustands diagnose, für Czern yKeller ist die Toxikose eine pathogenetische Definition.

Neuerdings ist Finkelstein von seiner ursprünglichen Auffassung abgerückt und hat sich dem Standpunkt Czerny - Kellers genähert, indem er die akuten dyspeptischen Störungen, bei denen das Fieber rein ,alimentär" bedingt ist, als leichte bzw. rudimentäre Toxikosen bezeichnet, und in der Intoxikation als schwerer Toxikose nur eine Steigerung erblickt, wobei er die früher geforderte Vollständigkeit der genannten Symptome aufgibt. Zu gleicher Zeit stellt Finkelstein die Intoxikation aber auch als Symptomenkomplex dar, der sich als eine besondere Reaktionsform an mannigfache Schädigungen verschiedenster Ätiologie anschließen kann; hier stellt er die Unabhängigkeit von der primären Schädigung als besonders beachtenswert hin. Der Unterschied zwischen Finkelstein und Czerny-Keller in bezug auf die Begriffsfassung der Toxikose besteht also zurzeit nur darin, daß ersterer die sog. rudimentären Toxikosen enger begrenzt sehen will, und zwar auf diejenigen akuten dyspeptischen Erkrankungen, in deren Mittelpunkt das alimentäre Fieber steht.

Uns erscheint es zunächst unzweckmäßig, die Genese der lokalen Darmerkrankung als toxisch zu bezeichnen. Der Begriff toxisch ist sehr verschwommen und kann je nach der persönlichen Einstellung zu einer einseitigen Auslegung führen. Wenn Czern y - Keller die Ansicht vertreten, daß in der Mehrzahl aller Fälle die Zersetzung der Nahrung bereits vor ihrer Verabreichung an die Kinder erfolgt, und die Häufung der Fälle in der heißen Jahreszeit eben durch diese Nahrungszersetzung bedingt ist, scheint uns ihre Annahme ebenso unbewiesen wie der von statistischer (Meinert) Seite diskutierte Zusammenhang, dessen hypothetischen Charakter Czerny - Keller verurteilen. Sprechen doch gerade die neueren Ernährungsversuche dafür, daß die Produkte der Milchzersetzung, die ja auch Czerny und Keller überwiegend nur in den Säuren sehen, nicht als die primär schädigenden 
Agentien bezeichnet werden können, die die Benennung der Erkrankung als Toxikose rechtfertigen.

Wenn man die Arbeit von Hamburger über die Behandlung der akuten Toxikosen mit Coliserum heranzieht, in der er berichtet, daß bei der üblichen diätischen Therapie in der Czernysehen Klinik 95\% starben, so wird man wohl daraus schließen dürfen, daß auch Czerny in der Praxis den Begriff der. Toxikose enger faßt, als dies nach seiner Definition erwartet werden muß; denn bei der geschilderten Fassung dürfte diese hohe Mortalität kaum verständlich sein. Es geht hieraus am besten die Unzweckmäßigkeit einer unscharfen Begriffseinstellung hervor.

Auch ältere Auffassungen legten die Deutung nahe, daß die Intoxikation das Resultat einer Steigerung der beim Enterokatarrh bzw. bei der Dyspepsie wirksamen Schädigung ist. Dies trifft aber generell nicht zu. Es entzieht sich nach unserer Erfahrung vorläufig völlig der klinischen Beurteilung, welche Fälle zur Intoxikation neigen. Nicht selten besteht bei erkrankten Kindern ein starkes Mißverhältnis zwischen der Schwere der lokalen Darmerkrankung und dem allgemeinen Vergiftungsbild. Damit ist nahegelegt, daß die Schädigung, die die Intoxikation auslöst, nicht mit der Ursache der Dyspepsie identifiziert werden darf. Sie verläuft von ihr unabhängig. Vermutlich spielt der individuelle Faktor, begründet in irgendeiner konstitutionellen Disposition (acidotische Konstitution?) oder Kondition eine maßgebende, aber vorläufig noch nicht klar erkennbare Rolle. Die Intoxikationsschwelle ist individuell verschieden. Viele Atrophiker zeigen unabhängig vom Grade der chronischen Störung auch trotz schwerer akuter Störungen keine Intoxikationssymptome. Die Intoxikation ist also nur eine Reaktionsform, und es ist zweckmäßig, dieser Tatsache durch die Bezeichnung Rechnung zu tragen und von toxischer - nach unserer Auffassung besser von komatöser - Reaktionsform einer Ernährungsstörung zu sprechen. Die Darstellung von Finkelstein ließ hoffen, daß die von $\mathrm{ihm}$ vorgenommene Umschreibung des Zustandes einem therapeutischen Bedürfnis genügen würde. Finkelstein sagte: Die Intoxikation erheischt Hunger, Nahrungsaufnahme verschlechtert sie, bestimmte Nahrungsformen lösen sie geradezu aus. Der experimentelle Teil über die Auslösung von Intoxikationsformen ist nicht nachgeprüft, der praktische Teil der Beweisführung erscheint uns heute nicht mehr voll überzeugend. Hunger bessert sicher in einem Teil der Fälle die Bewußtseinstrübung, doch führt er durchaus nicht zuverlässig zur Heilung. Sieht man die praktischen Ergebnisse durch, findet man die Behandlungserfolge doch recht wenig befriedigend, sie werden um so ungünstiger, je schärfer die Begrenzung erfolgt. Die Nahrungsentziehung kann die Schädigung vermindern, durch die die 
der Begriffe Toxikose und Indoxikation als Krankheitsbezeichnung. 317

komatöse Reaktion ausgelöst wird, und dadurch die Heilung begünstigen. Sie ist aber kein spezifisches Heilmittel; und während der Prozentsatz der Heilungen selbst bei schweren dyspeptischen Zuständen ein ziemlich günstiger ist, ist er bei der Intoxikation außerordentlich ungünstig.

So wenig bestritten werden soll, daß der komatöse Zustand aus einer schrittweisen Entwicklung von Symptomen entstehen kann, so wenig wird unter praktischen Gesichtspunkten das Bedürfnis nach einer erweiterten Fassung zugegeben werden können. Alle als Initialsymptome bezeichneten Erscheinungen können, aber brauchen nicht zur Ausbildung des Koma zu führen, und so kann ihre Zusammengehörigkeit mit dem gleichen Recht bestritten werden, wie sie bewiesen erscheint. Das gilt für die Lactosurie ebenso wie für das alimentäre Fieber, für die Albuminurie ebenso wie für den Gewichtssturz.

Die Verwertung des Atemtypus bereitet besondere Schwierigkeiten. Finkelstein hat die große Atmung unter die Kardinalsymptome aufgenommen. Seine Umschreibung zeigt aber, daß er unter großer Atmung etwas anderes versteht als jenen Atmungstypus, den die Klinik der Acidose mit der Bezeichnung ,große Atmung" belegt. Fin kelstein charakterisiert vielmehr den tatsächlich häufig festzustellenden Typus der beschleunigten und vertieften Atmung und seiner Varianten. Die toxische Reizung des Atemzentrums führt in der Regel zu einer vertieften und verlangsamten Atmung; die Beschleunigung, die Finkelstein hervorhebt, ist zunächst nur Fieberatmung. Nur die Vertiefung kann für die Intoxikationscharakterisierung verwertet werden.

Der Reinheit der Deutung stehen aber noch weitere Schwierigkeiten entgegen. Es ist bei einem großen Teil dieser Fälle bereits eine pneumonische Erkrankung in der Entwicklung, die meisten intoxizierten Kinder sterben mit einer Bronchopneumonie. So muß in der Bezeichnung des veränderten Atemtypus als toxische Atmung eine gewisse Zurückhaltung walten. Nur die Formen, die tatsächlich der großen Atmung entsprechen, also die vertiefte und verlangsamte Atmung, dürften verwertbar sein. Es genügt hier zu zeigen, daß die Verwertung des Atemtypus allein noch nicht zur Stellung der Diagnose führt.

So bleibt doch nur die Bewußtseinstrübung, die die Charakterisierung der toxischen Reaktion sichert und die. Therapie beeinflußt. Alles weitere ist Beiwerk. Dem ärztlichen Blick bleibt es überlassen, diese Trübung in frühestem Stadium in der charakteristischen Äußerung von Mimik und Bewegungen zu erkennen.

Jedenfalls wäre es zweckmäßig und würde das Verständnis erleichtern, wenn in Úbernahme der Gepflogenheiten der inneren Medizin nicht das Endsymptom zur Bezeichnung eines Krankheitsbildes verwendet würde, wie das beim Begriff der Intoxikation bzw. der Toxikose geschieht. 
318 L. Langstein u. H. Langer: Bedenken geg. d. Begriffe d. Toxikose u. Toxikation.

Man sollte den Dyspepsiebegriff beibehalten, durch den der Blick auf den Ausgangspunkt des pathologischen Vorgangs gelenkt wird, wenn man nicht zu der älteren, durch die neuere Forschung gerechtfertigten, Bezeichnung Enterokatarrh zurückgreifen will, durch die die Schädigung des Nährdarms deutlich zum Ausdruck kommt. Jedenfalls gleichgültig, welche Bezeichnung man für die primäre Krankheit wählen will, für die komatöse Ausgangsform ist eine begrifflich abtrennende selbständige Bezeichnung unberechtigt und unzweckmäßig. Die Bezeichnungals Dyspepsiemit drohenderoder manifester komatöser Reaktion würde eine Verständigung innerhalb der pädiatrischen Literatur auch ohne ausführliche Krankengeschichten ermöglichen und dem didaktischen Zweck die eindringlichste Begriffsform bieten. Darüber hinaus würde aber eine solche voraussetzungslose Terminologie auch die Verständigungsmöglichkeit mit anderen Disziplinen erleichtern.

Berlin, Kaiserin Auguste Victoria-Haus. 